\title{
Das Werk Alexander Kluges lesen/schauen/hören/ spüren. Audiovisuelle Montagen als Movens eines ästhetischen Bildungsprojekts
}

Lire/regarder/entendre/sentir l'ouvre d'Alexander Kluge. Les montages audiovisuels comme motivations d'un projet d'éducation esthétique

Reading/Viewing/Hearing/Feeling Alexander Kluge's work. Audiovisual montage as movens of an aesthetical educational project

\section{Florian Wobser}

\section{OpenEdition}

Journals

Édition électronique

URL : http://journals.openedition.org/ceg/1205

DOI : $10.4000 /$ ceg. 1205

ISSN : 2605-8359

Éditeur

Presses Universitaires de Provence

Édition imprimée

Date de publication : 18 décembre 2015

Pagination : 153-164

ISBN : 979-1-03200-020-5

ISSN : 0751-4239

\section{Référence électronique}

Florian Wobser, « Das Werk Alexander Kluges lesen/schauen/hören/spüren. Audiovisuelle Montagen als Movens eines ästhetischen Bildungsprojekts », Cahiers d'Études Germaniques [Online], 69 | 2015, Online erschienen am: 17 Dezember 2017, abgerufen am 26 November 2020. URL : http:// journals.openedition.org/ceg/1205; DOI : https://doi.org/10.4000/ceg.1205 


\section{Das Werk Alexander Kluges lesen/schauen/hören/spüren. Audiovisuelle Montagen als Movens eines ästhetischen Bildungsprojekts}

Florian WOBSER

Universität Rostock

[für chinchi]

\section{„Is that all there is?" (Peggy Lee)}

Reading/Viewing Alexander Kluge's Work lautete der Titel einer internationalen und transdisziplinären Konferenz ${ }^{1}$, der auf die mannigfaltigen Produktionen des Denkers und Künstlers Kluge und auf die variierend-korrelierenden Weisen ihrer Rezeption anspielt - und doch nicht genügt. Blickt man auf diesen Titel, bleibt unklar, ob Lesen/Schauen ein Kontrast, verknüpfender Chiasmus oder Ausdruck der „Philosophie des Dazwischen“" 2 ist. Ruft man sich aber Kluges Werk in Erinnerung, wird schnell klar, dass dessen Formen sich nicht lediglich zwischen Lesen/Schauen einordnen lassen. Die Literatur Kluges fordert zwar gewiss eine Lektüre und seine Kinofilme müssen angeschaut werden - doch letztere würden ohne das Hören ihrer Tonspuren partiell rezipiert und für einige der Texte Kluges gilt, dass eingefügte Bilder, Diagramme etc. betrachtet und gedeutet (d.h. lediglich im weitesten Sinne „gelesen") werden müssen. Dass Kluges Werk nicht allein zu lesen bzw. zu schauen, sondern genauso zu hören und sogar zu spüren ist, soll im Folgenden aber weder an Literatur noch an Filmen Kluges, sondern mittels seiner mit Oskar Negt gestalteten Philosophie und seiner bis heute für das (Web-)TV produzierten Kulturmagazine erläutert werden.

\footnotetext{
1 Gregory Cormann, Jeremy Hamers und Céline Letawe danke ich für ihre Mühe bei der Organisation der Konferenz - meine Kritik an deren Titel sei mir bitte verziehen.

2 Vgl. Rainer Stollmann, ,Vernunft ist ein Gefühl für Zusammenhang“, in Christian Schulte/Rainer Stollmann, Der Maulwurf kennt kein System. Beiträge zur gemeinsamen Philosophie von Oskar Negt und Alexander Kluge, Bielefeld, transcript, 2005, S. 233-267 (speziell S. 234).
} 
Verknüpft werden Theorie und massenmediale Praxis Kluges - in meiner „Lektüre“ - im Montageprinzip, das Prozesse einer Medialität antreibt, die aisthetisch erscheinen, wobei Kluges TV-Sendungen (seit 1988) als multisensorische Radikalisierungen des schon in Geschichte und Eigensinn (1981) unorthodoxen performativen Stils von Kluge/Negt gedacht werden. Diese massenmediale Medialität ist zwischen Öffentlichkeit und Erfahrung (1972) bzw. Maßverhältnisse des Politischen (1992) konzipiert worden und wird hier als ein Bildungsprojekt charakterisiert (2). Ich folge dabeimeiner These, dass diese, ,gemeinsame Philosophie“ Kluge/Negts ein solches Bildungsprojekt für den ,unterschätzten Menschen“" ${ }^{3}$ stets intendiert hatte, aber erst in der Medienpraxis Kluges konsequent Ausdruck erhält. Die „Gegen-Verwirklichung“ ${ }^{4}$ Kluges Denkens in/mittels der televisuellen Sphäre soll durch Bezüge auf kritische Theoretiker aus Frankfurt (3) und Frankreich (4) plausibel werden. Dieses ,philosophische Dazwischen“ werde ich als eine „Dialektik der Signifikanz" mit dem Wandel Kritischer Erziehungswissenschaft zu Modellen ästhetisch-performativer Bildungsprozesse verbinden (5) und an- und abschließend auch deren philosophiedidaktisches Potenzial kurz andeuten (6). Kluges WerkFragmente müssen als exemplarisch, gar als singulär begriffen werden. Indem ich pars pro toto inhaltliche sowie formale Zusammenhänge ${ }^{5}$ zwischen jenem - hier wortwörtlich - Opus magnum namens Geschichte und Eigensinn und dem audiovisuellen Essay Die Sahara wurde Sumpfgebiet (erstgesendet im Jahre 2000) aufzeige, werde ich gleichwohl meinen alternativen Titel dieses Beitrags Das Werk A. Kluges lesen/schauen/hören/spüren legitimieren.

\section{„There's more to life than this!““ (Björk)}

Kluge/Negt zielen früh auf eine emanzipatorische massenmediale Praxis ab, mit der die „Gebrauchswerteigenschaft“ von Öffentlichkeit und Erfahrung beachtet wird und die ihrer kritischen Prämisse folgt, nach der bestehendes TV „nur durch andersgeartete Produktionen“" zu verändern sei. ${ }^{6}$ Solch innovative „Gegenproduktionen“ werden von Kluge/Negt in ihrer späten Sammlung in Maßverhältnisse des Politischen eingeordnet, worin jede und jeder subtiles Unterscheidungsvermögen benötige. ${ }^{7}$ Es werde u.a. durch erneutes und erneuerndes „Lesenlernen“ erworben und ist

3 Vgl. Alexander Kluge/Oskar Negt, Der unterschätzte Mensch. Gemeinsame Philosophie in zwei Bänden, Frankfurt am Main, Zweitausendeins, 2001.

4 Ich argumentiere dafür, dass Kluge - im deleuzianischen Sinne - als ein vielseitiger „Akteur demnach das Ereignis [verwirklicht]“. Vgl. Gilles Deleuze, Logik des Sinns, Frankfurt am Main, Suhrkamp, 1993, S. 186-192 (speziell S. 188).

5 Dass Zusammenhang hier als Zusammenhänge beansprucht wird, greift nicht diese terminologische Kategorie an; es zeigt sich darin jedoch ein gewisses Misstrauen gegen deren Gewalt (vgl. Alexander Kluge/Oskar Negt, Geschichte und Eigensinn. Frankfurt am Main, Zweitausendeins, 1981, S. 777-1089).

6 Vgl. hierzu Alexander Kluge/Oskar Negt, Öffentlichkeit und Erfahrung. Zur Organisationsanalyse von bürgerlicher und proletarischer Öffentlichkeit, Frankfurt am Main, Suhrkamp, 1972, S. 20 bzw. S. 220 (für die letzten zwei Zitate).

7 Vgl. hierzu insgesamt Alexander Kluge/Oskar Negt, Maßverhältnisse des Politischen. 15 Vorschläge zum Unterscheidungsvermögen, Frankfurt am Main, Fischer, 1992. 
so das rezeptive Pendant des frühen Appells. Kluge/Negt bilden ein theoretisches framing, in dessen Rahmen ihr praxisorientierter Wunsch televisueller Gestaltungen auf Erfordernisse adäquaten Wahrnehmens in der umfassenden Medialität trifft. Kluge denkt Realität als Textur; anzustreben sei darin die Ausbildung im Lesen des Textes wirklicher Verhältnisse bzw. im „Nachlesen von Erfahrung“ aus „Übereinanderschreibungen“. 8

Dass Kluge/Negt unter Beachtung ihrer theoretischen und technischen Gegenwart jederzeit implizit den Zusammenhang zwischen Mensch und Welt als „höchste und proportionierlichste Bildung seiner Kräfte zu einem Ganzen“" ${ }^{\text {" }}$ beschreiben wollen, wird explizit bestätigt. Sie gestehen früh ein, dass sie u.a. „Ansprüchen traditioneller Bildung, Erziehung durch Wissenschaft, Autonomie, Reflexion auf Sinn u.s.w“ ${ }^{\text {10 }}$ verpflichtet seien, um später einer politischen Bildungspraxis Ausdruck zu verleihen:

Soweit sich die Elemente und Quellen des Politischen fassen lassen, haben sie ihre Kraft vor allem in den Formen. Die politischen Energien und Qualitäten brauchen Zeit, erkennbare Orte, Autonomiefähigkeit der Subjekte, einschließlich einer glücklichen Verbindung von Spontaneität und Dauer, ein gegenständliches Gegenüber (Reibungsfläche), den freien Wechsel zwischen Rückzug (Schlaf, Pause, Entlastung) und der Konzentration der Kräfte (Solidarität, Schutz, Wachheit) u.v.m. ${ }^{11}$

Im Zentrum der Publikationen Kluge/Negts steht jedoch ihr Opus magnum, worin sie phylo- und ontogenetische Bildungsprozesse im Spannungsfeld aus Geschichte und Eigensinn verorten. Der emanzipatorische Eigensinn soll zwischen den Antagonismen aus „lebendiger gegen entfremdete Arbeitskraft“ bzw. dem „Lustgegen das Realitätsprinzip“ wirken. ${ }^{12}$ Für Kluge/Negt sind kritische Anschlüsse an Marx und an Freud allgemein von hoher Relevanz; hier führen deren Einflüsse speziell zur Aussage über die Poetologie des eigenen Philosophierens:

Dieses Spielerische der Philosophiearbeit - gerade die kritische nimmt daran teil - ist die Bedingung für die ,Verflüssigung“ [...] der autoritativen Anordnung der Dinge [...]. Die Arbeitsweise der Sinne antworten darauf. Es gibt einen materialistischen Instinkt, dieses übermächtige Realitätsgebilde zu anarchisieren, das Übermächtige in Witz zu zerlegen. Wenn Brecht [...] Dialektik als ,Witz der Sache“ definiert, dann ist dieser Witz [...] Entdeckungen der Disproportionen in der Sache selber. Es ist eine elementare Wahrnehmungsweise: Komik, freie Assoziation, Erinnern, Antizipieren. Der Gegensatz heißt Schreckensstarre, Fixierung, Gefühlsballung, Dummsein. ${ }^{13}$

8 Vgl. ibid. S. 193-229 (speziell S. 229; S. 198 und 220 [für die letzten drei Zitate]).

9 Vgl. Wilhelm v. Humboldt, ,Ideen zu einem Versuch, die Gränzen der Wirksamkeit des Staats zu bestimmen“, in Werke I, Darmstadt, Wissenschaftliche Buchgesellschaft, 1980, S. 64.

${ }^{10}$ Kluge/Negt, Öffentlichkeit, S. 154.

11 Ibid.

${ }_{12}$ Vgl. hierzu vor allem Kluge/Negt, Geschichte, S. 98f. bzw. S. 48f., 212f., 335f., 375f. und $421 \mathrm{f}$.

13 Ibid., S. 96f., Anm. 9. 
Die Fußnote zu Theorie/Praxis der „Verflüssigung“ hat programmatischen Charakter. In ihr wird die aus einem Zusammenprall von Marx/Freud im Werk Kluge/Negts resultierende Strategie klar. Letztere zitieren in allen drei Schriften den frühen Topos von Marx, nach welchem die „Sinne daher unmittelbar in ihrer Praxis Theoretiker geworden [sind] " ${ }^{14}$, um hiermit auch entfesselte Phantasie nach Freud als „lebendige Arbeit des Moments“"15 zu verknüpfen. Von intellektuellen Grabenkämpfen ihrer Zeit unbeeindruckt stellen Kluge/Negt gleichzeitig Bezüge zu Vertretern der Avantgarde (z.B. Brecht), der „Frankfurter Schule“ (Benjamin, Adorno, Habermas) und - recht früh - der Dekonstruktion (Derrida) bzw. des Poststrukturalismus (Deleuze/Guattari) her. Den gleich zu Beginn der KlugeRezeption gewählten Topos der "Gesamtmontage"16 übernehme ich für diese schwierig zu vermittelnden Bezüge und deute sie - im Sinne meiner These - in der Fluchtlinie des hierdurch konzipierten Bildungsprojekts.

Kluge strebt sowohl für Print-Publikationen als auch für audiovisuelle Produktionen die maximale Formenvielfalt an. In allen sich sukzessiv etablierenden Massenmedien könne, Gegengiftnur inmitten dieser Entwicklung, an den Nahtstellen“ (z.B. zwischen den dominanten und alternativen Wissenschaftspraktiken bzw. TVÖffentlichkeiten) Wirkung entfalten. Die institutionell subversive Taktik der suture betont Kluge auch als ästhetische. Bereits in Geschichte und Eigensinn hatten die „Agents provocateurs“ des marxistischen Diskurses nonchalant Textformate, Bilder, Diagramme etc. eingefügt. Das Montageprinzip erhält in ihrem „Gebrauchsbuch“ ${ }^{17}$ also in Form uneindeutiger Text-Bild-Relationen performativ Ausdruck und provoziert die „Lektüre“, in der z.B. zur Beziehungsarbeit in Privatverhältnissen präsentativ-bildlich sowie diskursiv-skriptural hineinmontierte Informationen zu im Eis verspielt herumrutschenden Ottern in den eigensinnigen Bildungsprozess $\mathrm{zu}$ integrieren sind. Im Film sieht Kluge zugleich ein Potenzial zur medialen Radikalisierung zugunsten der wirklichkeitskonstituierenden Sinnlichkeit bzw. Phantasie, sobald er betont, dass

die Kinoprojektion auf einer Belichtung von $1 / 48$ Sekunde [beruht], der eine Dunkelphase
von 1/48 Sekunde folgt [...]. Das Auge sieht 1/48 Sekunde nach außen und 1/48 Sekunde
nach innen. Die Wirkungen der Filmmontage sind durch diese Pausen erst möglich. Die
Information steckt bei Montagewirkungen weder in der ersten noch in der folgenden
Abbildung, sondern beruht auf nachwirkenden Bildern, im Idealfall auf ,ungesehenen“
Bildern, die aufgrund der Differenz, der Lücke in der Information kontrastreicher Bilder
als Epiphanie entstehen.

${ }_{14}$ Vgl. zu diesem Zitat jeweils Kluge/Negt, Öffentlichkeit, S. 486, Anm. 3; Kluge/Negt, Geschichte, S. 931 und Kluge/Negt, Maßverhältnisse, S. 138.

15 Negt/Kluge, Geschichte, S. 938.

${ }_{16}$ Vgl. bereits früh Rainer Lewandowski, Alexander Kluge, München, Beck, 1980, speziell S. 50-59 bzw. zuletzt Bernd Stiegler, „Die Realität ist nicht genug. A. Kluges praktische Theorie und theoretische Praxis der Montage“, Text + Kritik, 85/86, Alexander Kluge, 2011.

17 Kluge/Negt, Geschichte, S. 5.

18 Alexander Kluge, „Die Macht der Bewußtseinsindustrie und das Schicksal unserer Öffentlichkeit“, in Klaus von Bismarck, Industrialisierung des Bewußtseins. Eine kritische Auseinandersetzung mit «neuen» Medien, München u.a., Piper, 1985, S. 121f. (für die letzten zwei Zitate). 
Der Film inkorporiert jetzt also Kluge/Negts Satz vom eingeschlossenen Dritten und ihr Prinzip Hautnähe ${ }^{19}$ und wird zu einer „Filmleinwand als Haut, die mit der Netzhaut Kontakt hält". Die Filmmedien sind die Membran, mit der ,das ES direkt [spricht]“. ${ }^{20}$ Der ästhetische Eigensinn eines Films fordert performativ den seines Publikums heraus - jenes „Dazwischen“, diese Medialität selbst wird zum diskontinuierlichen Prozess eines permanenten audiovisuellen Montierens. Dessen radikale „Verflüssigung“ ist nicht allein retrospektiv Modell für die Produktionen zugunsten von Öffentlichkeit und Erfahrung, sie dient ferner ebenso als mediale Form der Maßverhältnisse des Politischen, in denen „, der Begriff des Politischen den äußersten Intensitätsgrad einer Verbindung oder Trennung, einer Abstoßung oder Anziehung, einer Assoziation oder Dissoziation[beschreibt]: in jedem Zusammenhang möglich und in jeder Eigenschaft, die erfahrungsfähig ist, aktualisierbar". ${ }^{21}$

\section{„I've seen it all... I've seen it all...“ (Björk)}

Nimmt man dieses massenmediale Montageprinzip Kluges im - hier relevanten - bildungsphilosophischen focus ernst, so ist dieses nicht allein geeignet, sein Projekt genauer zu charakterisieren, sondern auch die theoretischen Bezüge von Kluge/Negt zu pointieren. Das Montieren ist vor allem eine künstlerische Praxis der Avantgarde; philosophisch steht die neuartige Erfahrungen fordernde Strategie quer zu Habermas' sprachpragmatisch-diskursethischer Theorie; Adorno kritisiert sie in Hauptwerken ${ }^{22}$, lässt aber „die Bedeutung von Montage und Verfremdung gegenüber Realismus“ in Erziehung zur Mündigkeit entgegen seiner sonstigen Skepsis medienpädagogisch gelten. Er gesteht ein, dass im TV immer jedes „Mittel des Schocks [...] wirksamer ist als die Gewöhnung“"23 und ist Benjamin hier ungewöhnlich nahe. Letzterer hatte Montage jederzeit bejaht. Im Kunstwerk-Aufsatz betont er, dass die ,physische Schockwirkung“ montierter Filme als „starke taktile Qualität“" eine ,gesteigerte Geistesgegenwart“ bei den Rezipienten auslöse. ${ }^{24}$ Dieser praktische Effekt der Montage ist Benjamin auch theoretisch im Passagen-Werk ein Vorbild. Dort montiert er fremde Zitate zur eigenen Textur und unterstreicht explizit

19 Vgl. Kluge/Negt, Geschichte S. 42f. bzw. S. 287-293.

${ }^{20}$ Alexander Kluge, ,Authentizität“ bzw. „Thesen 1-4“, in In Gefahr und größter Not bringt der Mittelweg den Tod, Berlin, Vorwerk 8, 2011, S. 131 bzw. S. 144 (für die letzten zwei Zitate).

${ }^{21} \mathrm{Negt} / \mathrm{Kluge}$, Maßverhältnisse, S. 91. Das Zitat im Zitat verweist zugleich auf die durch Kluge/ Negt intendierte „Verflüssigung“ C. Schmitts, wodurch sie Vertreter des frühen Links-Schmittianismus sind; vgl. hierzu etwa Oliver Marchart, Die politische Differenz, Berlin, Suhrkamp, 2010, S. 38ff.

${ }_{22}$ Zugunsten negativ-dialektischer Vermittlung kritisiert Adorno - in Briefen auch direkt Benjamin noch spät die Montage und bevorzugt einen Begriff ästhetischer Konstruktion, vgl. Theodor W. Adorno, Ästhetische Theorie, Frankfurt am Main, Suhrkamp, 1993, S. 72f. bzw. $90 \mathrm{f}$.

${ }^{23} \mathrm{Vgl}$. Theodor W. Adorno, „Fernsehen und Bildung“, in Erziehung zur Mündigkeit, Frankfurt am Main, Suhrkamp, 1982, S. 67f.

${ }^{24}$ Walter Benjamin, „Das Kunstwerk im Zeitalter seiner technischen Reproduzierbarkeit“, 3. Fassung, in Gesammelte Schriften I.2, Frankfurt am Main, Suhrkamp, 1991, S. 502f. (für die letzten drei Zitate). 
die ,[p]ädagogische Seite des Vorhabens“, indem er präsentativ wie diskursiv das „bildschaffende Medium“ des Menschen erregen und dialektisch rückkoppeln will: „Bild ist die Dialektik im Stillstand“. ${ }^{25}$

Schließt man Benjamins visuelle, auditive und taktile „Dialektik im Stillstand“ mit dem Prinzip der Montage kurz, so ergeben sich einmal mehr ästhetischperformative Effekte. Die „Verflüssigung“ Kluges trifft paradoxerweise auch jeden „Stillstand der Dialektik“:

Das Zusammentreffen von sprachlichen, akustischen und visuellen Formen und ihrer Integration in der Montage macht den Film zu komplexeren Aussagen fähig [...]. Im Film verbinden sich radikale Anschauung im visuellen Teil und Begriffsmöglichkeiten in der Montage zu einer Ausdrucksform, die ebenso wie die Sprache ein dialektisches Verhältnis zwischen Begriff und Anschauung ermöglicht, ohne daß dieses Verhältnis wie in der Sprache stabilisiert ist. ${ }^{26}$

Jedes multisensorische Bild wird in zwei konträre Richtungen verschoben/ verdichtet, dennMontierenist Trennenund Verbindenzugleich.Dieseepistemologische Spannung appelliert an sich Bildende, entzieht sich aber diskursiver dialektischer Vermittlung und ist als eine präsentative - so meine weitere These - ebenfalls mit dekonstruktiven bzw. poststrukturalistischen Mitteln treffend nachzuzeichnen.

\section{.and I have it all here - in red, blue, green..." (Radiohead)}

Wenn Kluge durch seine „montage sauvage“ ${ }^{\text {27 }}$ auf eine multisensorische und instabile Sprache zielt, liegt es nahe, zu deren adäquater Beschreibung radikal signifikante und dynamische Modelle zu wählen. Es bietet sich die „flottierende“ und „supplementäre“ Bewegung der différance Derridas an. ${ }^{28}$ Die Kraft dieses dekonstruktiven Herzstücks kann einerseits als Dynamisierung signifikanter Spuren und so als das „Trennende“ im „Dazwischen“ des Montierens gelten. Derrida überrascht - im Gespräch mit Stiegler ${ }^{29}$ über Massenmedien - sogar durch die Beanspruchung quasi-sinnlicher Phänomene, wenn er zur „freien Entfaltung der schöpferischen Produktion“ u.a. „das Timbre der Stimme, das Bild, den Blick,

${ }^{25}$ Vgl. hierzu Walter Benjamin, „Das Passagenwerk“, Gesammelte Schriften V, S. 571 (hier für die ersten zwei Zitate) bzw. S. 577.

${ }^{26}$ Alexander Kluge/Wilfried Reinke/Edgar Reitz, „Wort und Film“, in Klaus Eder/Alexander Kluge, Ulmer Dramaturgien. Reibungsverluste, München/Wien, Hanser, 1980, S. 16 [Hervorhebungen F. W.].

${ }^{27}$ Vgl. hierzu erstmals Werner Barg, „Ein Dokumentarist des Protestes. Alexander Kluges Theorie des Dokumentarfilms. Beobachtungen zu seinen Essay-Filmen und Fernsehmagazinen“, in Manfred Hattendorf, Perspektiven des Dokumentarfilms, München, Schaudig \& Ledig, 1995, S. 113.

${ }^{28} \mathrm{Zu}$ u.a. diesen Attributen der différance und ihrer Herleitung sei hier - viel zu pauschal verwiesen auf Jacques Derrida, „Die Struktur, das Zeichen und das Spiel im Diskurs der Wissenschaften vom Menschen“, in Die Schrift und die Differenz, Frankfurt am Main, Suhrkamp, 1976, S. $422-442$ sowie auf Jacques Derrida, „Die Différance“, in Randgänge der Philosophie, Wien, Passagen, 1988, S. 1-56.

${ }^{29}$ Derridas Dialogpartner Bernard Stiegler hat die Zusammenhänge um Öffentlichkeit und Erfahrung jüngst fortgesetzt; vgl. Bernard Stiegler, Logik der Sorge, Frankfurt am Main, Suhrkamp, 2008/2009. 
die Bewegung der Hände“ zählt und für das Kino- bzw. TV-Bild ebenfalls einen medienpädagogischen Imperativ formuliert:

\begin{abstract}
Wir glauben im ersten Moment von einem Bild als umfassender und unanalysierbarer, unzerlegbarer Gesamtheit überwältigt zu werden. Aber [...]: Man kann Bilder zerschneiden, von Sekunde zu Sekunde in Fragmente zerlegen [...]. Man muß lernen, Bilder wahrzunehmen, zu unterscheiden, zu komponieren, Collagen zu bilden, mit einem Wort zu montieren. ${ }^{30}$
\end{abstract}

Das „Verbindende“ im Montageprozess wiederum soll nicht mit Derrida, sondern mit Deleuze/Guattari erfasst werden, um zuletzt ebenso „Transzendenz“ und „Immanenz“ zu verknüpfen. ${ }^{31}$ Deleuze/Guattari denken immanent im Modus des agencement und erfinden die Begriffe Ritornell und Rhizom, die als Ereignisse variierende asignifikante Expressivitäten als „Schwingung mit unendlich vielen Obertönen oder enthaltenen Vielfachen, wie eine Klang- oder Lichtwelle“ figurieren. ${ }^{32}$ Die den „Denkakt" und die „Sensibilia“ einende „Heterogenese“, in deren multisensorisch-transdimensionaler Prozessualität jedoch „der Begriff bereits ins ausgeschlossene Dritte entwichen ist“", bündeln Deleuze/Guattari schließlich zur Antwort auf die Frage Was ist Philosophie?. Sie sei „die Kunst der Bildung [...] von Begriffen“" und impliziere auch ,eine Pädagogik des Begriffs“"33, die Deleuze in seinen zwei Kino-Büchern noch um eine ,,audiovisuelle Pädagogik“ ${ }^{34}$ erweitert. Letztere sei u.a. durch die Nouvelle Vague initiiert worden und gilt ebenso für Kluges TV, das er in Nachfolge Godards konsequent als Vertreter der „Partei des Kinos“ konzipiert. ${ }^{35}$

Hatte schon Benjamin den ,zerstreuten Examinator" ${ }^{36}$ des modernen Films bildlich als „,Kaleidoskop, das mit Bewußtsein versehen ist““, bezeichnet und betont, dass dieses zwecks seiner Befreiung, ,zerschlagen werden [muß]“37, so denken Deleuze/Guattari audiovisuell-taktile Ausdrucksintensitäten als ein „RaumZeit-Kristall“, der im Kino als Zeit-Bild „denkendes Bild“ werde, um einen „Schock im Denken entstehen zu lassen“"38. In diesem „Denk-Schock-Fluss“ zwischen Frankfurt und Frankreich stehen auch die TV-Montagen Kluges. Dessen (Fake-) Interviews könnten als sensomotorische Affekt-Bilder beschrieben, seine radikal Zitate).

${ }^{30}$ Vgl. Jacques Derrida/Bernard Stiegler, Echographien, S. 59, 50 und 156 (für die letzten drei

31 Vgl. Giorgio Agamben, „Die absolute Immanenz“, in Bartleby oder die Kontingenz gefolgt von Die absolute Immanenz, Berlin, Merve, 1998, S. 125f.

$32 \mathrm{Vgl}$. zu den Begriffen des Ritornells bzw. Rhizoms Gilles Deleuze/Félix Guattari, Tausend Plateaus, Berlin, Merve, 2005, S. 423-479 bzw. S. 11-42 sowie Gilles Deleuze, Die Falte, Leibniz und der Barock, Frankfurt am Main, Suhrkamp, 2012, S. 128 (für das Zitat).

${ }^{33}$ Vgl. Gilles Deleuze/Félix Guattari, Was ist Philosophie?, Frankfurt am Main, Suhrkamp, 2000, S. 27f. (für die ersten zwei Zitate); S. 9, 29f., 6 [Hervorhebung F. W.] und 17 (für die folgenden Zitate).

34 Vgl. hierzu Gilles Deleuze, Das Bewegungs-Bild. Kino 1, Frankfurt am Main, Suhrkamp, 1997, S. 27f. bzw. Gilles Deleuze, Das Zeit-Bild, Kino 2, Frankfurt am Main, Suhrkamp, 1991, S. $316 f$.

${ }^{35}$ Vgl. Kluge, „Bewußtseinsindustrie“, S. 75. Diesem konzeptionellen Transfer soll in diesem Beitrag ohne Beachtung technisch-medialer Veränderungen von (analogem) Kino zu (digitalem) TV gefolgt werden.

${ }^{36}$ Vgl. Benjamin, „Kunstwerk“, S. 505.

37 Vgl. hierzu Walter Benjamin, ,Charles Baudelaire. Ein Lyriker im Zeitalter des Hochkapitalismus“, in Gesammelte Schriften I.2, S. 630 bzw. S. 660.

38 Vgl. Deleuze/Guattari, Plateaus, S. 476 bzw. Deleuze, Zeit-Bild, S. 134 und S. 205. 
„verflüssigten“ audiovisuellen Essays aber sollen hier im Sinne Deleuzes - und als „Gegen-Verwirklichung“ von Geschichte und Eigensinn - als kristallines Zeit-Bild$T V$ charakterisiert werden.

\section{,....and I try and I try and I try...“ (Rolling Stones)}

Blickt man auf Anfang/Ende von Hegels Gang der Begriffsarbeit, so ist sie flüssig. ${ }^{39}$ Kluge will durch massenmediale Montagen aber nicht bloß Kants transzendentale Ästhetik intensivieren, sondern durch „Verflüssigung“ gar Hegels Dialektik überbieten. Darum spreche ich bei Kluge - mittels einer zwei getrennte Paradigmen montierenden Begriffserfindung - von einer Dialektik der Signifikanz. Kluge gelingen audiovisuell ästhetische-performative Radikalitäten, die durch maximale „Verflüssigung“ nahezu gasförmige Wolken produzieren. ${ }^{40}$ Die vom Montageprinzip hergeleiteten Bezüge auf Derrida bzw. Deleuze/Guattari mögen unter Beachtung des beiläufigen Zitierens des Ersteren bzw. Kritisierens Letzterer in Geschichte und Eigensinn übertrieben wirken. ${ }^{41}$ Doch lassen spätere Aussagen von Kluge/Negt deren Legitimierung zu. Beide seien laut Negt durch „Derrida [...] im Sinne unserer Kritik eines verengten Modernitätsbegriffs “ 42 geprägt worden; Kluge bejaht allgemein, dass Kritik für Unterscheidungsvermögen „eine schöne Übersetzung [wäre], weil es ja eigentlich Unterscheidungsvermögen heißt. Difference auf Französisch“, betont an anderer Stelle viel spezieller: „Dieses sich wandelnd Lebendige ist ein ,Rhizom', nach einem Ausdruck von Guattari und Deleuze, also unwillkürlicher Zusammenhang“ und erklärt gar das „Kristallgitter“ zur filmtheoretischen ,idée fixe“, worin „Zusammenhang [kristallisiert]“. ${ }^{43}$

Der audiovisuelle Essay Kluges mit dem Titel Die Sahara wurde Sumpfgebiet ${ }^{44}$ (ca. 15') - für diesen Beitrag wiederum zur Analyse ausgewählt - gewährt seinen Rezipienten als - s.o. - massenmediales „Gegenüber“ eine ästhetisch-performative „Reibungsfläche“.

39 Vgl. Werner Stegmaier, Art. „Fließen“, in Ralf Konersmann (Hrsg.), Wörterbuch der philosophischen Metaphern, Darmstadt, Wissenschaftliche Buchgesellschaft, 2007, S. 102-121 (speziell S. 106f. bzw. S. 110f.).

${ }^{40}$ Zum Ziel des gasförmigen Schreibens, vgl. Gilles Deleuze, Unterhandlungen 1972-1990, Frankfurt am Main, Suhrkamp, 1993, S. 195 bzw. zur Singularität ,als Wolke“ Deleuze, Falte, S. 101.

${ }^{41} \mathrm{Vgl}$. Kluge/Negt, Geschichte, S. 375-413 und S. 1062f. bzw. S. 294f., 486f. und 753f. Der Kurzschluss zwischen Marx und Derrida zur neuen Tanzmelodie (vgl. S. 398f., Anm. 23) könnte wiederum zur Politik der Differenz (vgl. Anm. 20) samt „Marx’ Gespenstern“ (Derrida) in Beziehung gesetzt werden.

${ }^{42}$ Vgl. Oskar Negt u.a., „Der Maulwurf kennt kein System. O. Negt im Gespräch mit R. Stollmann und C. Schulte“, in Schulte/Stollmann, Der Maulwurf, S. 13.

${ }^{43} \mathrm{Vgl}$. Alexander Kluge, Verdeckte Ermittlung. Ein Gespräch mit C. Schulte und R. Stollmann, Berlin, Merve, 2001, S. 49; Alexander Kluge, „Der Autor als Dompteur oder Gärtner. Rede zum HeinrichBöll-Preis 1993“, in Personen und Reden, Berlin, Wagenbach, 2012, S. 38 (für die ersten zwei Zitate) und Alexander Kluge, „Interview von Ulrich Gregor“, Mittelweg, S. 200 [alle Hervorhebungen F. W.].

${ }^{44}$ Vgl. Alexander Kluge, „Die Sahara wurde Sumpfgebiet“, in Seen sind für Fische Inseln. Fernseharbeiten 1987-2008, DVD 8.1, Frankfurt am Main, Zweitausendeins, 2009 bzw. die hiervon leicht abweichende Version unter www.dctp.tv/filme/die-sahara-wurde-sumpf/ [02/01/2015]. 
Ihre „Lektüre“, die aufgrund vieler Inserts zwischen Lesen/Schauen changieren muss, konstituiert sich aber zumindest - s.u. - auch durch das Hören. Kluges Essay besitzt Intro (ca. 3') und Outro (ca. 1,5'), die den Hauptteil einpassen. Der gesamte Beitrag widmet sich der Kontingenz der Zeit, die ein- und ausleitend durch Animationen zu dem Raum des Alls in Relation gesetzt und deren Relativität früh mit popkulturellen Clips und Schrifttafeln anhand schwankender Zeit-Modi unter Einfluss von Lysergsäure-diethylamid erläutert wird. Subjektive Zeitschrumpfung und Zeitsprengung wird also visuell nach innen/außen suggeriert und findet auditiven Ausdruck, indem sich u.a. kosmisch-kreisende World-, dynamisch-drängende Techno- und experimentelle Klänge abwechseln. Im Mittelteil lassen Inserts mit aleatorischen Daten aus früher Zeit die menschliche Evolution/Historie linear und doch zufällig wirken. Montiert sind dazwischen einige Bildsequenzen, die weder szientifisch noch künstlerisch erscheinen oder in der sich selbstreflexiv die eigene Ästhetik zeigt.

Dieser Essay gewinnt durch die Strategie der „Verflüssigung“ nicht allein seine Form, sondern auch seine Thematik. Gegenüber Kluges „kristallisiertem Zusammenhang“ aus radikal montierten diskursiven/präsentativen Formelementen stürzt aber jede „Lektüre“ in die Krise. Genau solch „ein krisenhaftes Ereignis“ und dessen Überschreiten stellt wiederum der Bildungstheoretiker Hans-Christoph Koller ins Zentrum seiner Theorie transformatorischer Bildungsprozesse. Er spitzt Humboldts Reflexionen $\mathrm{zu}$ heterogenen Sprachvermögen $\mathrm{zu}$ und erläutert, dass Bildung geschieht, sobald neue „Dispositionen der Wahrnehmung, Deutung und Bearbeitung von Problemen“ aktiviert werden und jeweilige Verhältnisse zur Welt, zu Anderen bzw. sich selbst innovieren. ${ }^{45}$

In einem phänomenologischen Zusammenhang nutzt Koller die Modelle negativer und fremdartiger Erfahrung ${ }^{46}$ dazu, ästhetische Krisen zu beschreiben und hermeneutische bzw. dialektische Ansätze zu erweitern. Um sie fortzuschreiben, soll auf die Krise eine Antwort erfolgen, die eine andere Ordnung produziert und statt zu einer „Horizontverschmelzung“ (Gadamer) zu einem „Wandel“" eines individuellen Horizonts führt. ${ }^{47}$ Zur Ermöglichung dieser resoluten „Verknüpfung unsres Ichs mit der Welt zu der allgemeinsten, regesten und freiesten Wechselwirkung“"48, greift Koller dann ebenso auf dekonstruktive bzw. poststrukturalistische Taktiken zurück. Er betont, dass Bildung, ,sich im Medium der Sprache an und mit Subjekten vollzieht“ und beruft sich u.a. auf Derridas ,signifikante Bewegung“ als partikulares Pendant einer jeden „Totalität der ,Erfahrung““ und Movens der Entstehung neuer Lesarten. ${ }^{49}$

${ }^{45} \mathrm{Vgl}$. Hans-Christoph Koller, Bildung anders denken. Einführung in die Theorie transformatorischer Bildungsprozesse, Stuttgart, Kohlhammer, 2012, S. 15f. (für die letzten zwei Zitate).

${ }^{46}$ Vgl. ibid. S. 71-78 (zu G. Buck) bzw. S. 79-86 (zu B. Waldenfels).

47 Zum Umgang mit Horizontverschiebungen vgl. wiederum auch Kluge/Negt, Geschichte, S. 1004-1089.

${ }^{48}$ Humboldt zitiert nach Koller, Bildung, S. 11.

49 Ibid. S. 95 [Hervorhebung F. W.] und S. 128 (für die letzten zwei Zitate) sowie insgesamt S. $122-135$. 
Koller gehört zu einer Gruppe einiger Bildungstheoretiker, die einen cut gegenüber Kritischer Erziehungswissenschaft präferieren. ${ }^{50}$ Er schließt nicht mehr an Adorno oder z.B. Andreas Gruschka ${ }^{51}$ an, seine phänomenologische Subversion der Dialektik ist der „Dialektik im Stillstand“ Benjamins bzw. Kluges „Dialektik der Signifikanz“ nahe und das ästhetisch-performative Theorem des „Transformatorischen“ der „Verflüssigung“ besonders affin. Während Koller sich auf Butlers Praxis der Resignifizierung beruft, um Erfahrung und diskursive Konstruktionen miteinander zu verknüpfen, wird dieser Schritt hier - im Sinne Kluges - mittels der Deleuze folgenden Bildungsphilosophie von Olaf Sanders unternommen.

Sanders kann in seiner Einzelstudie zu Deleuzes Pädagogiken genauer als Koller auf seine(n) Referenzautor(en) eingehen ${ }^{52}$ und bietet mit Blick auf Kluge einen Vorteil: über die diskursive hinaus bezieht er eine präsentative Performativität mit ein. Er sieht in Deleuze/Guattaris Begriffsbildungen und Bildungsprozesslogik, aber ebenso in Deleuze' Kinobildung die „doppelte Artikulation“ wirksam. ${ }^{53}$ Dieses transzendental/empirische ${ }^{54}$ Faktum zeige sich aktuell/virtuell in Konzepten und Perzepten und sei sich ereignender präsentativ/diskursiver Ausdruck. Sanders radikalperformative Bildungsphilosophie schließt die Krise und die Entstehung des Neuen permanent kurz. Mit Hinweis auf den Appell von Deleuze, dass wir, ,in einer neuartigen Weise das Visuelle lesen und den Sprechakt auf neue Art hören müssen“, fordert Sanders, dass kritische Dialektik von ästhetisch-performativer Bildungsprozesslogik abzulösen sei: „Bildung lässt sich als ein Prozess von Kristallbildung begreifen“. 55 Solche „verflüssigend-transformatorischen“ Bildungsprozesse werden auch für den „doppelt artikulierten“ audiovisuellen Parforce-Ritts Kluges durch Evolution/ Historie und dessen Zeitsprengung notwendig.

\section{„love makin' on screen - impossible dream“ (Radiohead)}

An letztgenannter Stelle behauptet Sanders ebenso: „Unterricht funktioniert anders“. Der unwissende Lehrmeister ${ }^{56}$ dient ihm zur Warnung, dass gerade eine

\footnotetext{
${ }^{50}$ Vgl. hierzu z.B. Markus Rieger-Ladich, Mündigkeit als Pathosformel. Beobachtungen zur pädagogischen Semantik, Konstanz, UVK, 2002.

51 Vgl. in der Nachfolge Adornos Andreas Gruschka, Negative Pädagogik. Einführung in die Pädagogik mit Kritischer Theorie, Wetzlar, Büchse der Pandora, 2004.

${ }_{52}$ Zur Koller-Kritik Sanders - der selbst aber Guattari bloß im Untertitel nennt - vgl. Olaf Sanders, „Philosophie pädagogisieren. Lyotard zum Beispiel“ - und „Wozu Bildungsphilosophie?“, in Alfred Schäfer/Christiane Thompson, Pädagogisierung, Halle-Wittenberg, MLU, 2013, S. 85-102, www. pedocs.de/volltexte/2013/7722/pdf/Schaefer_Thompson_2013_Paedagogisierung.pdf [03/01/2015].

53 Vgl. insgesamt Olaf Sanders, ,Deleuzes Pädagogiken. Die Philosophie von Deleuze und Deleuze/ Guattari nach 1975“, Manuskript [erscheint 2015 im Katzenberg-Verlag in Hamburg]; speziell zu der „,doppelten Artikulation“ vgl. S. 24f., S. 32f. bzw. S. 94f. (Begriffsbildung) und S. 367-387 (Kinobildung). 2012.

54 Vgl. hierzu Marc Rölli, Philosophie des transzendentalen Empirismus, Wien u.a., Turia + Kant,

55 Vgl. Deleuze, Zeit-Bild, S. 316 bzw. Sanders, „Pädagogiken“, S. 148.

56 Vgl. Jacques Rancière, Der unwissende Lehrmeister. Fünf Lektionen über die intellektuelle Emanzipation, Wien, Passagen, 2009.
} 
Erziehung zur Mündigkeit zu falschen Anschlüssen ${ }^{57}$ führe. Diese Gefahr ist ernst zu nehmen; ich bin aber überzeugt, dass unter Beachtung der Theorie Kluges auch seine Medienpraxis zur mediensensibilisierten Bildung im Schulunterricht ihren Unort besitzen sollte. In Unzeiten permanenter Krisen und beschleunigter Bildungsprozesse gilt es, sie als „Gegengift“ zu nutzen und Kluges Geständnis „Es gibt eine Politik der Schulstunde und es gibt eine Politik der Schulpause. Bei mir geht es um die Schulpause“" 58 - für die Stärkung von Phantasie, Eigensinn bzw. Unterscheidungsvermögen Heranwachsender zu wenden. Das Wahrnehmen von und Konstruieren heterogenen Sinns zu Die Sahara wurde Sumpf ist eine „Verknüpfung unsres Ichs mit der Welt“ unserer massenmedialen „Jetztzeit“" (Benjamin) und fachdidaktisch zu initiieren. ${ }^{59}$

Didaktiken des Philosophierens als Kulturtechnik und ihrer „Kompetenzorientierung " ${ }^{60}$ sind dafür medienphilosophisch auszulegen. Der formale Umgang mit audiovisuellen Medien ist in der Schule noch immer zweitrangig und kann mit phänomenologischen/dekonstruktiven Akzenten so geleistet werden, dass eine „negative Didaktik“ oder ein bloßes „Wahrnehmung wahrnehmen“ übertroffen wird. ${ }^{61}$ Es sollte mit Schülern zunächst das genaue Beschreiben dieses Essays Kluges eingeübt werden, wozu ggf. auch Reduktionen auf einzelne der Inserts, Bilder, Sequenzen etc. erlaubt scheinen. Die Schriftbildlichkeit ${ }^{62}$ mancher Inserts, selbstreferenzielle/ verweisende Bilder, die uneindeutigen Illustrationen, der irritierende Ausflug in die Comic-Fiktion oder das - klanglich betonte - bizarre Montage-Bild, das Kluges Montage-Ästhetik - die im Transfer zu erörtern wäre - in nuce aufzeigt, böten sich an. Die wechselhafte Musik sollte in allen Varianten hinzugezogen und genauso analytisch methodisch beachtet werden.

In thematischer Hinsicht - immer an die formale Gestaltung gekoppelt könnte die Konzentration auf dem Essay-Hauptteil liegen. Einzelne Daten wären von Schülern auf ihre Relevanz zu prüfen und bezüglich des evolutionären bzw. historischen Zusammenhangs zu erörtern (es bliebe den Schülern überlassen, Affirmation oder Kritik zu üben). Die alle betreffende Ereignishaftigkeit wäre dagegen fortzuschreiben, indem Mitglieder einer Lerngruppe z.B. einen eigenen Zeitstrahl der Phylo- oder ihrer Ontogenese gestalteten (freigestellt wären ihnen alle produktions-/ handlungsorientierten diskursiven/präsentativen Mittel).

${ }^{57}$ Vgl. hierzu Deleuze, Zeit-Bild, S. 168ff. bzw. Sanders, „Pädagogiken“, S. 362ff.

58 Zitiert nach: Jürgen Kaube, „Ein Ingenieur seiner Geschichten“, in FAZ, 11.09.2009.

59 Vgl. auch Florian Wobser, „Audiovisuelle Essays von Alexander Kluge im Philosophieunterricht - ,Verflüssigung ' des Zusammenhangs Geschichte-Gesellschaft-Geltung“, Münster, 2014, online unter: http://nbn-resolving.de/urn:nbn:de:hbz:6-12319383449 bzw. Florian Wobser, „,Vermutlich gibt es Parallel-Universen“ - Audiovisuelle Essays Alexander Kluges im Philosophieunterricht“, Hanno Depner, Band zur Tagung Visuelle Philosophie [Rostock, 2013, im Erscheinen].

${ }^{60} \mathrm{Vgl}$. Ekkehard Martens, Methodik des Ethik- und Philosophieunterrichts. Philosophieren als elementare Kulturtechnik, Hannover, Siebert, 2003 bzw. Johannes Rohbeck, Didaktik der Philosophie und Ethik, Dresden, Thelem, 2008.

${ }^{61}$ Vgl. Marion Pollmanns, Didaktik und Eigensinn. Zu A. Kluges Praxis und Theorie der Vermittlung, Wetzlar, Büchse der Pandora, 2006 bzw. Stephan Brössel, „Rätselhafte Reizüberflutung. A. Kluges Fernsehformate und die Reflexion von Wahrnehmung“, Der Deutschunterricht 2/2012, S. 78-87.

${ }^{62} \mathrm{Vgl}$. hierzu Eva Cancik-Kirschbaum/Sybille Krämer/Rainer Totzke, Schriftbildlichkeit. Wahrnehmbarkeit, Materialität und Operativität von Notationen, Berlin, Akademie Verlag, 2012. 
Den Abschluss der Beschäftigung mit Kluges Essay könnte nicht zuletzt der Transfer auf die abstrakte Kategorie Zeit bilden. Dieser hätte wiederum gewiss viele Facetten $^{63}$ - denn wer ist hinsichtlich der Zeit kein „unwissender Lehrmeister“?

\section{„Immerse your soul in love!“ (Radiohead)}

Dass Kluges auf dem Montageprinzip beruhende Theorie und seine massenmediale Praxis radikale Philosophie des Dazwischen - u.a. zwischen Frankfurt/Frankreich - und mehr als zu lesen/schauen ist, sollte in diesem Beitrag deutlich geworden sein. Dass das Dazwischen Kluges als ästhetisch-performatives bildungsphilosophisch und didaktisch relevant ist, wurde durch Koller/Sanders, mit deren Hilfe „Verflüssigung“ zum „doppelt artikulierten Transformatorischen“ wird, plausibel gemacht. Zum Prozess eines Sich-Bildens zählen alle Sinne - dass Film/Video neben ihrer Audiovisualität zusätzlich immerhin auch die Taktilität affizieren, gehört wiederum zum Phantasma zwischen Benjamins „Leibraum“, Derridas „paradoxer Verleiblichung“ sowie Deleuze/Guattaris die Differenz Materialität/Medialität suspendierendem „Expressiv-Werden“. Als Phantasma einer „Gegen-Verwirklichung“ - eines Versuchs, mittels Immersion der suture dieses „Realitätsgebilde zu anarchisieren“, ist Kluges „Dialektik der Signifikanz“ zu lesen/ schauen/hören/spüren.

${ }^{63}$ In älteren Jahrgängen müssten - vgl. das Intro - auch die Verschiebungen/Verdichtungen der Zeit durch Intoxikationen nicht ausgespart bleiben; vielmehr könnte dies ein sehr relevantes Thema sein. 